\title{
Statistical evaporation of rotating clusters. IV. Alignment effects in the dissociation of nonspherical clusters
}

\author{
P. Parneix \\ Laboratoire de Photophysique Moléculaire*, CNRS, \\ Fédération de recherche Lumière Matière, Bât. 210, \\ Université Paris-Sud, F91405 Orsay Cedex, France \\ F. Calvo \\ Laboratoire de Physique Quantique, IRSAMC, Université Paul Sabatier, \\ 118 Route de Narbonne, F31062 Toulouse Cedex, France
}

\begin{abstract}
Unimolecular evaporation in rotating, non-spherical atomic clusters is investigated using Phase Space Theory in its orbiting transition state version. The distributions of the total kinetic energy release $\varepsilon_{\mathrm{tr}}$ and the rotational angular momentum $J_{r}$ are calculated for oblate top and prolate top main products with an arbitrary degree of deformation. The orientation of the angular momentum of the product cluster with respect to the cluster symmetry axis has also been obtained. This statistical approach is tested in the case of the small 8-atom Lennard-Jones cluster, for which comparison with extensive molecular dynamics simulations is presented. The role of the cluster shape has been systematically studied for larger, model clusters in the harmonic approximation for the vibrational densities of states. We find that the type of deformation (prolate vs. oblate) plays little role on the distributions and averages of $\varepsilon_{\operatorname{tr}}$ and $J_{r}$ except at low initial angular momentum. However, alignment effects between the product angular momentum and the symmetry axis are found to be significant, and maximum at some degree of oblateness. The effects of deformation on the rotational cooling and heating effects are also illustrated.
\end{abstract}

\section{INTRODUCTION}

Statistical theories of unimolecular dissociation ${ }^{1.2}$ provide invaluable information about binding energies, lifetimes, and more generally the thermodynamical state of atomic and molecular clusters ${ }^{3}$ Besides their common use in helping to interpret experiments, ${ }^{4.5}$ their quantitative relevance has been demonstrated in numerical simulations ${ }^{6.7}$ with great

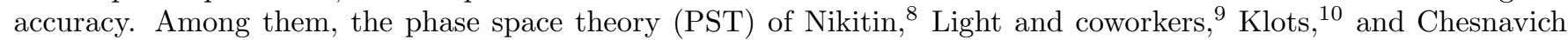
and Bowers ${ }^{11}$ stands out as the most successful ${ }^{6.7}$ in describing evaporation of weakly bound clusters for which the loose transition state hypothesis is satisfied. Within the PST formalism, statistical dissociation is characterized by the vibrational densities of states (VDOS) of the parent and product clusters, as well as the rotational density (RDOS) of the products. This latter quantity depends on the interaction between the fragments, and more importantly on

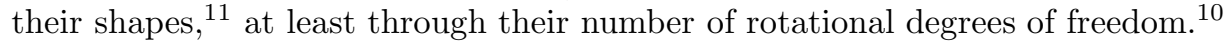

Large clusters of simple materials, such as rare gases or alkali metals, are usually found to be rather spherical. Small aggregates can nevertheless exhibit significant deformations at the scale of a few tens of atoms or molecules. The shape of covalent clusters could be inferred from the ion mobility measurements performed by the Jarrold group $\stackrel{12.13 .14}{=}$ In silicon clusters, a prolate-oblate transition was evidenced around 20 atoms $\stackrel{12}{\underline{12}}$ Small carbon clusters show a competition between linear chains, rings and planar shapes $\stackrel{13}{\underline{13}}$ Larger carbon clusters can be arbitrarily prolate under the nanotube

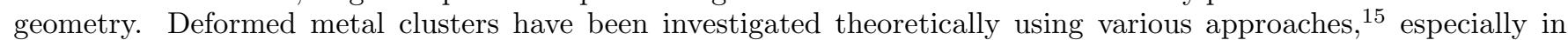
concern with the fission problem. 16

Accounting for deviations from the spherical shape may be crucial for calculating precisely the number of rovibrational states of small molecules, and the role of nonsphericity on the statistical observables relevant to unimolecular dissociation has been discussed previously. Chesnavich and Bowers $\stackrel{11.18}{18}$ quantified the error introduced in the rotational DOS when approximating symmetric rigid rotors by spherical tops, for small molecules or radicals with moderate deformations. Berblinger and Schlier ${ }^{17}$ used a Monte Carlo integration method to compute the density of quantum states of the oblate molecules $\mathrm{H}_{3}^{+}$or $\mathrm{HD}_{2}^{+}$using appropriate densities or rotational states.

Peslherbe and Hase considered evaporation in small aluminium clusters from the point of view of molecular dynamics (MD) simulations and PST: ${ }^{7}$ In this work, deformation effects were not fully accounted for, because the centrifugal barrier was neglected except in the spherical top approximation for the fragments. However it is known that centrifugal forces, and more generally rotation may affect cluster properties quite significantly, ${ }^{19,20.21}$

\footnotetext{
* laboratoire associé à l'université de Paris-Sud
} 
In our previous work, an extensive study of evaporation in rotating atomic and molecular clusters was carried out 22.23.24 paying a special attention to the final angular momentum and kinetic energy released. By carefully calculating the vibrational and rotational DOS's pertaining to various cases of spherical clusters emitting atoms, ${ }^{22.23}$ linear or spherical molecules $\underset{24}{2}$ we could extend the conclusion of Weerasinghe and Amar ${ }^{6}$ that PST is quantitative in reproducing the exact numerical results. However some clear deviations could be seen in the statistical treatment of evaporation when applied to the smaller cluster $\mathrm{LJ}_{8}$, where the main product $\mathrm{LJ}_{7}$ was approximated as a spherical top. This poorly satisfactory result was attributed to the non-spherical character of $\mathrm{LJ}_{7}$, known to be oblate in its most stable energy structure.

Our first goal in this paper is to incorporate the effects of deformations in the PST treatment for rotating clusters. As a first application, atomic dissociation in $\mathrm{LJ}_{8}$ will be considered as an example. More generally, we will focus on large clusters simplified as molten, homogeneous droplets, in order to investigate the role of nonsphericity on the observables relevant to cluster dissociation. Following previous effort,, 7.24 .25 it is hoped that cluster evaporation could be used as a probe of the shape, and possibly the phase changes, in the main product. The dynamical simulation of fragmentation in asymetric molecules such as those aforementioned involve can be very challenging from a computational point of view. Therefore it is important to incorporate and quantify the effects of deformation in the rate theories used to interpret gas-phase experiments on clusters.

In the next Section, we summarize the theoretical results of phase space theory in the approximation where the main product after atom dissociation can be modelled as a prolate or oblate top. Concise expressions for the rotational densities of states, KER and final angular momentum are proposed. We also compute the distribution of the relative orientation between the symmetry axis and the angular momentum in the product. This property, along with its average value, provide an estimation of how the rotational energy is distributed in the cluster. We illustrate the specific case of $\mathrm{LJ}_{8}$ and larger LJ clusters in Sec. III. In particular, the roles of size, total energy and angular momentum on the final properties are discussed. Finally, we summarize and give some concluding remarks in Sec. IV]

\section{METHODS}

\section{A. Phase Space Theory}

The rotational density of states (RDOS) is a key function within the framework of phase space theory ${ }^{8.9 .10 .11}$ This quantity depends on the kinetic energy released after dissociation, $\varepsilon_{\mathrm{tr}}$, sum of the translational and rotational parts. It also depends on the total angular momentum $J$. It is obtained by integration of the rotational sum of states $\Gamma\left(\varepsilon_{r}^{*}, J_{r}\right)$, which quantifies the number of rotational states available at a given angular momentum $J_{r}$ of the product cluster, and a kinetic energy lower than $\varepsilon_{r}^{*}$, its limiting value at the centrifugal barrier ${ }^{11}$

In PST the two fragments are treated as rigid bodies and the expressions of $\Gamma$ only depend on their corresponding symmetries. Our present interest concerns the evaporation from nonspherical atomic clusters, and we have chosen to deal with symmetric top deformations, prolate or oblate, but with an arbitrary degree of nonsphericity. The rotational constants are denoted as $A$ and $B$ for a prolate top cluster $(\lambda=A-B \geq 0)$, and as $B$ and $C$ for an oblate top cluster $(\lambda=B-C \geq 0)$. The rotational energy $\varepsilon_{r}$ is expressed by $\varepsilon_{r}=B J_{r}^{2} \pm \lambda K^{2}$, where $J_{r}$ and $K$ are the internal angular momenta, with + (resp. - ) for the prolate top (resp. oblate top) case.

One motivation of the present work is to investigate the possible correlations between the cylindrical symmetry axis of the main product cluster and the angular momentum vector after fragmentation. Therefore the most general statistical quantity we need to consider is the probability for a dissociation event to occur with prescribed values of $K, J_{r}$, and $\varepsilon_{\mathrm{tr}}$ :

$$
P\left(K, J_{r}, \varepsilon_{\mathrm{tr}} ; E, J\right) d K d J_{r} d \varepsilon_{\mathrm{tr}} \propto \omega\left(E-E_{0}-\varepsilon_{\mathrm{tr}}\right) d K d J_{r} d \varepsilon_{\mathrm{tr}} \int_{\mathcal{L}} d L
$$

where $\omega$ is the vibrational density of states of the product cluster, $E_{0}$ the energy difference between the parent and product, and $\mathcal{L}$ the available values of orbital momentum $L$ at fixed $J_{r}, \varepsilon_{\mathrm{tr}}$, and $K$. The RDOS results from summing the above equation over all $\left(K, J_{r}\right)$ values compatible with energy and angular momentum conservation. For this we first need to integrate over $K$ for rotational energies no larger than $\varepsilon_{r}^{*}{ }^{11}$ The expressions of these sums of states are given by

$$
\Gamma= \begin{cases}2 J_{r}=\Gamma_{\mathrm{S}}\left(\varepsilon_{r}^{*}, J_{r}\right) & \text { if } \varepsilon_{r}^{*} \geq A J_{r}^{2} \\ 2\left[\frac{\varepsilon_{r}^{*}-B J_{r}^{2}}{\lambda}\right]^{1 / 2}=\Gamma_{\mathrm{P}}\left(\varepsilon_{r}^{*}, J_{r}\right) & \text { if } A J_{r}^{2} \geq \varepsilon_{r}^{*} \geq B J_{r}^{2}\end{cases}
$$


for the prolate top+atom case, and by

$$
\Gamma= \begin{cases}2 J_{r}=\Gamma_{\mathrm{S}}\left(\varepsilon_{r}^{*}, J_{r}\right) & \text { if } \varepsilon_{r}^{*} \geq B J_{r}^{2} \\ 2 J_{r}-2\left[\frac{B J_{r}^{2}-\varepsilon_{r}^{*}}{\lambda}\right]^{1 / 2}=\Gamma_{\mathrm{S}}\left(\varepsilon_{r}^{*}, J_{r}\right)-\Gamma_{\mathrm{O}}\left(\varepsilon_{r}^{*}, J_{r}\right) & \text { if } B J_{r}^{2} \geq \varepsilon_{r}^{*} \geq C J_{r}^{2}\end{cases}
$$

for the oblate top+atom case.

To obtain the rotational densities of states at given $\varepsilon_{\mathrm{tr}}$ and $J$, the sums of rotational states must be integrated in the whole range $\mathcal{C}$ of available values of $J_{r}$ and $L$, accounting for conservation of total energy and angular momentum. The general schematic integration plot is represented in Fig. 1(a). Here $X$ and $Y$ are the two rotational constants of the product, such that $X>Y$, equal to $A$ and $B$ (resp. $B$ and $C$ ) in the prolate top (resp. oblate top) case. The barrier height corresponding to orbital momentum $L$ is denoted as $\varepsilon^{\dagger}(L)$ in what follows. The procedure used to calculate the RDOS at finite angular momentum is basically the same as used previously by Chesnavich and Bowers, ${ }^{11}$ and by ourselves 22.24

The intersections $J_{r}(X)$ and $J_{r}(Y)$ have the respective values $\left(\varepsilon_{\operatorname{tr}} / X\right)^{1 / 2}$ and $\left(\varepsilon_{\operatorname{tr}} / Y\right)^{1 / 2}$. We introduce the limits $L_{X}^{-}$and $L_{X}^{+}$(resp. $L_{Y}^{-}$and $L_{Y}^{+}$) as the intersections of $\varepsilon_{\mathrm{tr}}=X J_{r}^{2}+\varepsilon^{\dagger}(L)\left(\right.$ resp. $\left.\varepsilon_{\mathrm{tr}}=Y J_{r}^{2}+\varepsilon^{\dagger}(L)\right)$ with $L=\left|J-J_{r}\right|$ (for $L_{X}^{-}$and $L_{Y}^{-}$) and with $L=J+J_{r}$ (for $L_{X}^{+}$and $L_{Y}^{+}$). At low $\varepsilon_{\mathrm{tr}}$, corresponding to $\varepsilon_{\mathrm{tr}} \leq \varepsilon^{\dagger}(J), L_{X}^{+}$and $L_{Y}^{+}$are also given from the intersections with $L=\left|J-J_{r}\right|$.

Moreover we must remind that when $J$ is not zero, the KER $\varepsilon_{\text {tr }}$ has a minimum value $\varepsilon_{\mathrm{tr}}^{\min }$ where integration starts. This value actually depends on the smallest rotational constants, namely $Y$. $\varepsilon_{\mathrm{tr}}^{\min }$ is such that the curve $\varepsilon_{r}^{*}=Y J_{r}^{2}$ is tangent to the line $L=J-J_{r}$.

The rotational sums of states, Eqn. (2) and (3), can be gathered into $\Gamma\left(\varepsilon_{r}^{*}, J_{r}\right)=\Gamma_{\mathrm{s}}\left(\varepsilon_{r}^{*}, J_{r}\right) \pm \Gamma_{\mathrm{t}}\left(\varepsilon_{r}^{*}, J_{r}\right)$, where $\Gamma_{\mathrm{s}}=2 J_{r}$ is the sphere+atom part, and $\Gamma_{\mathrm{t}}$ the non-spherical, symmetric top part. In the definition of $\Gamma$, a plus sign is used for the prolate top, and a minus sign for the oblate top. According to this simple partition, the RDOS can then be expressed as a sum of two terms:

$$
\begin{aligned}
\Gamma\left(\varepsilon_{\mathrm{tr}}, J\right) & =\iint_{\mathcal{C}} \Gamma\left(\varepsilon_{r}^{*}, J_{r}\right) d J_{r} d L \\
& =\Gamma_{\mathrm{s}}\left(\varepsilon_{\mathrm{tr}}, J\right) \pm \Gamma_{\mathrm{t}}\left(\varepsilon_{\mathrm{tr}}, J\right) .
\end{aligned}
$$

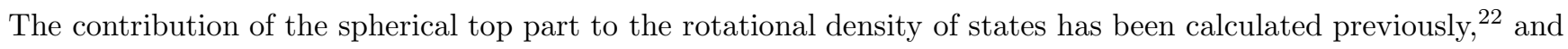
reads

$$
\Gamma_{\mathrm{s}}\left(\varepsilon_{\mathrm{tr}}, J\right)=\Gamma_{\mathrm{s}}^{-}+\Theta\left(\varepsilon_{\mathrm{tr}}-B_{s} J^{2}\right) \Gamma_{\mathrm{s}}^{+},
$$

where $\Theta$ is the Heaviside function, and where $B_{s}$ is the rotational constant equal to $A$ (prolate top) or $C$ (oblate top). The two components $\Gamma_{\mathrm{s}}^{-}$and $\Gamma_{\mathrm{s}}^{+}$of $\Gamma_{\mathrm{s}}$ are given by

$$
\Gamma_{\mathrm{s}}^{-}\left(\varepsilon_{\mathrm{tr}}, J\right)=\int_{L_{B_{s}}^{-}}^{L_{B_{s}}^{+}}\left[\frac{\varepsilon_{\mathrm{tr}}-\varepsilon^{\dagger}(L)}{B_{s}}-(J-L)^{2}\right] d L,
$$

and

$$
\Gamma_{\mathrm{s}}^{+}\left(\varepsilon_{\mathrm{tr}}, J\right)=2 J\left(L_{B_{s}}^{-}\right)^{2}
$$

respectively.

Similarly, the non-spherical RDOS $\Gamma_{\mathrm{t}}\left(\varepsilon_{\mathrm{tr}}, J\right)$ can be expressed as a sum of two terms, namely $\Gamma_{\mathrm{t}}=\Gamma_{\mathrm{t}}^{-}+\Theta\left(\varepsilon_{\mathrm{tr}}-\right.$ $\left.Y J^{2}\right) \Gamma_{\mathrm{t}}^{+}$. These two terms cannot be fully explicited in general, due to the unknown (or complex) dependence of $\varepsilon^{\dagger}$ or $\varepsilon_{r}^{*}$ with $L$. However, integral forms are readily available from

$$
\Gamma_{\mathrm{t}}^{-}\left(\varepsilon_{\mathrm{tr}}, J\right)=\int_{L_{Y}^{-}}^{L_{Y}^{+}} d L \int_{\max \left(|\mathrm{J}-\mathrm{L}|, \sqrt{\varepsilon_{\mathrm{r}}^{*} / \mathrm{X}}\right)}^{\left(\varepsilon_{r}^{*} / Y\right)^{1 / 2}} 2\left|\frac{\varepsilon_{r}^{*}-B J_{r}^{2}}{\lambda}\right|^{1 / 2} d J_{r}
$$

and

$$
\Gamma_{\mathrm{t}}^{+}\left(\varepsilon_{\mathrm{tr}}, J\right)=\int_{L_{X}^{-} \Theta\left(\varepsilon_{\mathrm{tr}}-X J^{2}\right)}^{L_{Y}^{+}} d L \int_{\max \left(|\mathrm{J}-\mathrm{L}|, \sqrt{\left.\varepsilon_{\mathrm{r}}^{*} / \mathrm{X}\right)}\right.}^{J+L} 2\left|\frac{\varepsilon_{r}^{*}-B J_{r}^{2}}{\lambda}\right|^{1 / 2} d J_{r} .
$$


The integrals over $J_{r}$ are solved to yield

$$
\begin{gathered}
\Gamma_{\mathrm{t}}^{-}\left(\varepsilon_{\mathrm{tr}}, J\right)=\frac{1}{\sqrt{B \lambda}} \int_{L_{Y}^{-}}^{L_{Y}^{+}} d L \varepsilon_{r}^{*}\left[\Psi\left(\sqrt{\frac{B}{Y}}\right)-\Psi\left(\max \left(|J-L| \sqrt{\frac{B}{\varepsilon_{r}^{*}}}, \sqrt{\frac{B}{X}}\right)\right)\right] \\
\Gamma_{\mathrm{t}}^{+}\left(\varepsilon_{\mathrm{tr}}, J\right)=\frac{1}{\sqrt{B \lambda}} \int_{L_{X}^{-} \Theta\left(\varepsilon_{\mathrm{tr}}-X J^{2}\right)}^{L_{Y}^{+}} d L \varepsilon_{r}^{*}\left[\Psi\left((J+L) \sqrt{\frac{B}{\varepsilon_{r}^{*}}}\right)-\Psi\left(\max \left(|J-L| \sqrt{\frac{B}{\varepsilon_{r}^{*}}}, \sqrt{\frac{B}{X}}\right)\right)\right]
\end{gathered}
$$

with the function $\Psi$ given by

$$
\Psi(x)=x \sqrt{1-x^{2}}+\sin ^{-1} x
$$

for prolate top fragments, and by

$$
\Psi(x)=x \sqrt{x^{2}-1}-\ln \left(x+\sqrt{x^{2}-1}\right)
$$

for oblate top fragments.

\section{B. Kinetic energy release distribution}

From the density of rotational states, the kinetic energy release distribution can be easily calculated from the usual relationship

$$
P\left(\varepsilon_{\mathrm{tr}} ; E, J\right) \propto \int_{\varepsilon_{\mathrm{tr}}^{\min }}^{E-E_{0}} \Gamma\left(\varepsilon_{\mathrm{tr}}, J\right) \omega\left(E-E_{0}-\varepsilon_{\mathrm{tr}}\right) d \varepsilon_{\mathrm{tr}}
$$

in which $\omega$ corresponds to the vibrational density of states of the product cluster.

\section{Product angular momentum distribution}

We keep the notations introduced previously for the rotational constants $B_{s}, X$ and $Y$, such that $\left(B_{s}, X, Y\right)=$ $(A, A, B)$ for the prolate top fragment, and $\left(B_{s}, X, Y\right)=(C, B, C)$ for the oblate top fragment. We also note $L_{X}=\left(\varepsilon^{\dagger}\right)^{-1}\left(\varepsilon_{\mathrm{tr}}-X J_{r}^{2}\right)$, where $\left(\varepsilon^{\dagger}\right)^{-1}$ is the reciprocal function of $\varepsilon^{\dagger}$.

The probability distribution of $J_{r}, P\left(J_{r} ; E, J\right)$ is obtained from integration of Eq. (1) over $K$ and $\varepsilon_{\text {tr }}$. This quantity has been investigated in details in our past work. ${ }^{23} \mathrm{~A}$ similar approach can be pursued here, after using the fact that the RDOS is the sum of the two contributions $\Gamma_{\mathrm{s}}$ and $\pm \Gamma_{\mathrm{t}}$. Hence the distribution $P$ can be written as $P=P_{\mathrm{s}} \pm P_{\mathrm{t}}$, where the plus (resp. minus) sign is used for the prolate (resp. oblate) top fragment. The expressions for the spherical and symmetric top contributions are given by

$$
\begin{aligned}
P_{\mathrm{s}}\left(J_{r} ; E, J\right)= & 2 J_{r} \int_{\varepsilon_{\mathrm{tr}}^{\min }}^{E-E_{0}} \Theta\left[L_{B_{s}}\left(\varepsilon_{\mathrm{tr}}, J_{r}\right)-\left|J-J_{r}\right|\right] \\
& \times \omega\left(E-E_{0}-\varepsilon_{\mathrm{tr}}\right)\left[\min \left(J+J_{r}, L_{B_{s}}\right)-\left|J-J_{r}\right|\right] d \varepsilon_{\mathrm{tr}}
\end{aligned}
$$

and

$$
\begin{aligned}
P_{\mathrm{t}}\left(J_{r} ; E, J\right)= & \frac{2}{\sqrt{\lambda}} \int_{\varepsilon_{\mathrm{tr}}^{\min }}^{E-E_{0}} \Theta\left[L_{Y}\left(\varepsilon_{\mathrm{tr}}, J_{r}\right)-\left|J-J_{r}\right|\right] \\
& \times \omega\left(E-E_{0}-\varepsilon_{\mathrm{tr}}\right) d \varepsilon_{\mathrm{tr}} \int_{\max \left(\left|J-J_{r}\right|, L_{X}\right)}^{\min \left(J+J_{r}, L_{Y}\right)}\left|\varepsilon_{r}^{*}-B J_{r}^{2}\right|^{1 / 2} d L .
\end{aligned}
$$




\section{D. $\cos \theta$ distribution}

The relative orientation of the angular momentum of the product cluster with respect to its symmetry axis can be calculated by integrating Eq. (1) over all variables except $K$. At given $\varepsilon_{\mathrm{tr}}, J_{r}$ and $L$, the number of available $K$ states depends on the values of $\varepsilon_{r}^{*}=\varepsilon_{\mathrm{tr}}-\varepsilon^{\dagger}(L)$ and $J_{r}$. In the prolate top case, there are two states available if $\varepsilon_{r}^{*} \geq A J_{r}^{2}$. If $A J_{r}^{2} \geq \varepsilon_{r}^{*} \geq B J_{r}^{2}$, there are also two states available provided that $|K| \leq K_{\max }=\left[\left(\varepsilon_{r}^{*}-B J_{r}^{2}\right) / \lambda\right]^{1 / 2}$, and zero otherwise. The relative orientation $\cos \theta=|K| / J_{r}$ must then satisfy

$$
\varepsilon_{r}^{*} \geq\left(A \cos ^{2} \theta+B \sin ^{2} \theta\right) J_{r}^{2} .
$$

The same ideas lead to the following condition for the oblate top case:

$$
\varepsilon_{r}^{*} \geq\left(C \cos ^{2} \theta+B \sin ^{2} \theta\right) J_{r}^{2} .
$$

The integration over the $\left(L, J_{r}\right)$ plane is now restricted, as indicated in Fig. 1(b), to include the above conditions. At a given $\cos \theta$, the intersection $J_{r}(\theta)$ with the $L=0$ axis is given by $J_{r}(\theta)=\left[\varepsilon_{\operatorname{tr}} /\left(B \sin ^{2} \theta+B_{s} \cos ^{2} \theta\right)\right]^{1 / 2}$ in general. This quantity continuously sweeps the $\left[J_{r}(X), J_{r}(Y)\right]$ interval.

Finally we get the probability density that the relative orientation between the angular momentum vector and the revolution axes has the value $\cos \theta$ within $d \cos \theta$ as

$$
\begin{aligned}
P(\cos \theta ; E, J) & \propto \int_{\varepsilon_{\mathrm{tr}}^{\min }}^{E-E_{0}} \omega\left(E-E_{0}-\varepsilon_{\mathrm{tr}}\right) d \varepsilon_{\mathrm{tr}} \times\left\{\iint_{\varepsilon_{r}^{*} \geq X J_{r}^{2}} 2 d L d J_{r}+\right. \\
& \left.+\iint_{X J_{r}^{2} \geq \varepsilon_{r}^{*} \geq Y J_{r}^{2}} 2 \Theta\left[\varepsilon_{r}^{*}-\left(B \sin ^{2} \theta+B_{s} \cos ^{2} \theta\right) J_{r}^{2}\right] d L d J_{r}\right\},
\end{aligned}
$$

valid for both types of deformations. These distributions, as well as the preceding probabilities of $\varepsilon_{\text {tr }}$ or $J_{r}$, must be calculated numerically in general. Ingredients other than the rotational densities, most importantly the vibrational densities of states and the centrifugal energies $\varepsilon^{\dagger}(L)$, are obtained from the same techniques described in our previous work 22

However, the general shape of the distributions can be inferred already at this stage. In the prolate top situation, $B_{s} \geq B$ and $P(\cos \theta)$ is maximal when $\cos \theta=0$, and minimal when $\cos \theta=1$. The reverse holds for the oblate top case. Therefore, $P(\cos \theta)$ is a decreasing (resp. increasing) function of $\cos \theta$ for prolate (resp. oblate) fragments. This means that the angular momentum gets more likely aligned with the principal axis with largest inertia. Thus it simply reflects the greater rotational stability of rigid bodies when their angular velocity is lower ${ }^{28}$

\section{RESULTS AND DISCUSSION}

The clusters we are interested in are Lennard-Jones clusters characterized by an arbitrary degree of deformation. The simplest model is that of an ellipsoid, whose axes ratio can take any real value. At a given (large) number of constituants $n$, the reasonable assumption of constant density implies that the volume of the cluster is constant, which allows us to define the equivalent spherical radius $R_{s}$ as $R_{s}=R_{0} n^{1 / 3}$, where $R_{0}$ plays the role of a lattice parameter. In practice, $R_{0}$ is taken to reproduce the rotational constant of the icosahedral 13-atom cluster.

The ellipsoidal shape of the cluster is continuously varied by keeping its volume constant. By denoting $a$ and $b$ the long parallel and short perpendicular axes of the ellipsoid, respectively, we quantify deformation in the cluster using $\gamma=(b-a) /(b+a)$. Equating $R_{s}^{3}$ to $a b^{2}$ yields

$$
\gamma=\frac{1-\left(a / R_{s}\right)^{3 / 2}}{1+\left(a / R_{s}\right)^{3 / 2}}
$$

As the cluster becomes increasingly prolate, $\gamma$ decreases and tends to -1 , which corresponds to a linear system. For oblate systems, $\gamma$ increases toward +1 , which corresponds to a planar system. The rotational constants corresponding to the deformation $\gamma$ are given by

$$
B_{s}=\frac{5}{4 n R_{s}^{2}}\left(\frac{1-\gamma}{1+\gamma}\right)^{2 / 3}=B_{0}\left(\frac{1-\gamma}{1+\gamma}\right)^{2 / 3}
$$

and

$$
B=2 B_{0}\left[\left(\frac{1-\gamma}{1+\gamma}\right)^{4 / 3}+\left(\frac{1+\gamma}{1-\gamma}\right)^{2 / 3}\right]^{-1}
$$




\section{A. $\quad \mathbf{L J}_{8} \longrightarrow \mathbf{L} \mathbf{J}_{7}+\mathbf{L J}$}

The dissociation of $\mathrm{LJ}_{8}$ has been considered first since it provides a good candidate to test the proposed formalism, the product cluster $\mathrm{LJ}_{7}$ being not perfectly spherical in its ground state geometry. For this system, the anharmonic VDOS and the effective dissociation potential were obtained from parallel tempering Monte Carlo and Wang-Landau simulations, respectively. The technical details can be found in our previous work ${ }^{22}$

To quantify the real extent of deformation in the vibrationally excited $\mathrm{LJ}_{7}$ cluster, we have analysed the thermal evolution of its rotational constants. In Fig 2 the average constants are plotted versus $T$. At $T=0$, the minimum energy configuration is the pentagonal bipyramid, an exactly oblate system for which $A=B \approx 0.17, C \approx 0.11$. These rotational constants yield a deformation index $\gamma \approx 0.3$, or $a / R_{s}=0.7$ with $R_{s}=0.55$. At higher temperatures, the cluster deviates more and more from the oblate shape, eventually becoming prolate. In order to get meaningful statistics of the dissociation process using standard molecular dynamics, we had to thermalize the parent cluster at rather high temperatures, typically above the melting point $(T \approx 0.2)$. The rotational constants used in the PST analysis were taken at this precise temperature, yielding $A \approx 0.18$ and $B=C \approx 0.11 \mathrm{LJ}$ units. These values give an equivalent deformation index $\gamma=-0.3$ in the ellipsoidal picture, or $a / R_{s}=1.5$. Interestingly, the double icosahedron $\mathrm{LJ}_{19}$ is more spherical than $\mathrm{LJ}_{7}(\gamma \approx-0.18)$, and remains so even above its melting temperature.

In Table \we have reported the values of $\left\langle\varepsilon_{\mathrm{tr}}\right\rangle$ and $\left\langle J_{r}\right\rangle$ obtained by MD simulations and by the PST descriptions in various rigid body approximations for $\mathrm{LJ}_{7}$. These results correspond to $E / n=1.2$ and $J=0,1,2$ and 3 (one LJ unit of angular momentum approximately equals $33 \hbar$ for argon). The data for $J=0$ is obtained using simplifications to Eqn. (14] 19), which arise due to the new restraint on the orbital momentum, namely $L=-J_{r}, 10.11 .23 .24$

Under these aforementioned conditions, increasing cluster deformation $(\gamma<0$ or $\gamma>0)$ mainly increases the product angular momentum, and marginally reduces the KER. The prolate top approximation performs significantly better than the simple spherical assumption in reproducing the MD results, but this is also true for the oblate top approximation.

The complete distribution of $J_{r}$ contains more information than the average value alone. This distribution is plotted in Fig. 3 for the initial angular momentum $J=3$. A good agreement between MD and PST is obtained when the deformation of the product cluster is taken into account. By comparison, the distribution in the spherical approximation underestimates $J_{r}$. More interestingly, the PST/oblate calculation looks very close to the prolate result, suggesting that the type of deformation does not alter significantly the rotational distribution after evaporation. The KER distribution, not plotted here, shows a similar behavior. However, it should be kept in mind here that we are dealing with only moderate deviations from sphericity.

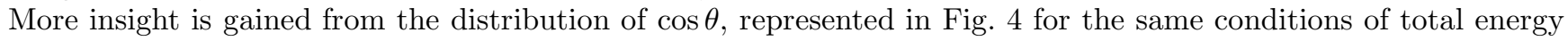
and angular momentum. The distribution is obviously uniform in the spherical case, as there is no privileged axis. As expected from our previous analysis, the prolate description of the product cluster favors low $\cos \theta$. This is in agreement with MD, but differs from the PST result in the oblate approximation.

Increasing angular momentum in the parent cluster brings the final rotation axis closer to the major principal axis, as shown in Fig. [5 This emphasizes the need for properly describing deformations in clusters having a significant or even moderate rotational motion. The values of $J$ considered in this figure are small enough to produce rotational heating $\stackrel{23.26}{2}$ However high angular momenta, which result in rotational cooling, also display a similar behavior. Comparing the values of $\langle\cos \theta\rangle$ at $J=0$ and $J=3$ shows that the deviation from random orientation (for which $\langle\cos \theta\rangle=1 / 2)$ is half due to the extent of deformation, and half due to angular momentum itself. From this figure, but also from Fig. [ alignment between the rotation axis and the main symmetry axis is about twice more efficient for prolate tops than for oblate tops. Such an effect is expected to be also magnified by the extent of deformation.

\section{B. Size effects in model clusters}

We now discuss the general effects of deformation on the statistical properties of larger clusters after evaporation. Our interest here is not to reproduce MD or experimental results, for this reason we have used an approximate model, where the $n$-atom LJ cluster is treated as a continuous medium with constant density, and the vibrational density of states is assumed to be harmonic. Such a model allows us to vary arbitrarily the degree of deformation $\gamma$ in the whole range $-1<\gamma<1$. The interaction potential is also simplified as $-4 n / r^{6}$, which yields a barrier energy $\varepsilon^{\dagger}$ proportional to $L^{3}$. As deformation of the cluster increases, the physical extent of the cluster also increases and we expect the $1 / r^{6}$ approximation to fail eventually. A more appropriate potential such as $1 /\left(r-r_{0}\right)^{6}$ would not be relevant for the present, qualitative discussion, even though it would provide a more robust ground for eventual comparisons.

Three parent cluster sizes $n+1$ have been selected, which correspond to $n=50,100$, and 200, respectively. To cover a vast range of situations, two total energies $(E / n=0.9$ and $1.2 \mathrm{LJ}$ units per atom) and two angular momenta $(J=0$ and $J=20$ LJ units) have been considered, thus providing four different physical conditions for fragmentation. The 
effects of deformation on the kinetic energy released and on the final angular momentum are shown in Figs. 6] and (7) respectively, for the four present situations. From a general point of view, smaller clusters show more pronounced deviations of the average KER, product angular momentum. This is easily understood by noticing that the rotational constants, hence all the parameters which characterize the deformation such as $\lambda$, decrease with size very fast $\left(\propto n^{-5 / 3}\right)$ with respect to, e.g. the centrifugal barrier energy $\left(\propto n^{-1 / 2}\right)$. In other terms, the specific symmetric top contributions $\gamma_{\mathrm{t}}$ to the RDOS become less important with increasing size.

It is useful to analyse first the $J=0$ case, since all the integrals involved are one-dimensional. For convenience, we assume in the following discussion that the main product is a prolate top. In this case the RDOS is exactly given for $J=0$ and a $C_{6} / r^{6}$ interaction potential by ${ }^{23}$

$$
\begin{aligned}
\Gamma\left(\varepsilon_{\mathrm{tr}}, J=0\right) & =\int_{0}^{J_{r}^{A}} 2 J_{r} d J_{r} \\
& +\int_{J_{r}^{A}}^{J_{r}^{B}} 2\left[\frac{\varepsilon_{\mathrm{tr}}-a J_{r}^{3}-B J_{r}^{2}}{\lambda}\right]^{1 / 2} d J_{r},
\end{aligned}
$$

where $a J_{r}^{3}=\varepsilon^{\dagger}\left(J_{r}\right)$ is the centrifugal energy at the barrier. Here $a$ does not depend on $\gamma$ but only on $n$ and $C_{6}$. $J_{r}^{A}$ and $J_{r}^{B}$ are solution of $\varepsilon_{\mathrm{tr}}=A J_{r}^{2}+a J_{r}^{3}$ and $\varepsilon_{\mathrm{tr}}=B J_{r}^{2}+a J_{r}^{3}$, respectively. When $a=0$, this integral can be exactly solved as

$$
\Gamma\left(\varepsilon_{\mathrm{tr}}, J=0\right)=\frac{\varepsilon_{\mathrm{tr}}}{\sqrt{B \lambda}}\left[\frac{\pi}{2}-\sin ^{-1} \sqrt{\frac{B}{A}}\right] .
$$

$\Gamma$ is a linear function of $\varepsilon_{\mathrm{tr}}$ independently of the rotational constants, therefore the average KER does not change with deformation, because of the normalization of the integrals involved in the average. This shows that any variation in the average energy released is due to the centrifugal barrier only. The Klots models 10 approximate the rotational densities $\Gamma\left(\varepsilon_{\mathrm{tr}}\right.$, low $\left.J\right)$ as a power law in $\varepsilon_{\mathrm{tr}}$, the exponent increasing proportionally with the number of rotational degrees of freedom. Since this number does not depend on the possible symmetry of the products, there are no deformation effects on the evaporation statistics within the Klots model.

We have not found simple expressions for the RDOS by Taylor expanding the general formula of Eq. (23) in $a$, because the result still contained complex functions of the rotational constants. Instead we consider the $J_{r}$ observable. The general expression for the probability distribution of $J_{r}$ at $J=0$, for prolate top product, reads ${ }^{23}$

$$
\begin{aligned}
P\left(J_{r} ; J=0\right) & =2 J_{r} \int_{A J_{r}^{2}+a J_{r}^{3}}^{E-E_{0}} \omega\left(E-E_{0}-\varepsilon_{\mathrm{tr}}\right) d \varepsilon_{\mathrm{tr}} \\
& +\int_{B J_{r}^{2}+a J_{r}^{3}}^{A J_{r}^{2}+a J_{r}^{3}} 2\left[\frac{\varepsilon_{\mathrm{tr}}-a J_{r}^{3}-B J_{r}^{2}}{\lambda}\right]^{1 / 2} \omega\left(E-E_{0}-\varepsilon_{\mathrm{tr}}\right) d \varepsilon_{\mathrm{tr}} .
\end{aligned}
$$

After some algebra, the lowest order in expansion in $|\gamma|^{1 / 2}$ of this expression is found to be

$$
\begin{aligned}
P\left(J_{r} ; J=0\right) & \simeq 2 J_{r} \int_{B_{0} J_{r}^{2}+a J_{r}^{3}}^{E-E_{0}} \omega\left(E-E_{0}-\varepsilon_{\mathrm{tr}}\right) d \varepsilon_{\mathrm{tr}} \\
& +2 \sqrt{2} B_{0}^{3 / 2}|\gamma|^{3 / 2} J_{r}^{4} \omega^{\prime}\left(E-E_{0}-B_{0} J_{r}^{2}-a J_{r}^{3}\right)
\end{aligned}
$$

where $\omega^{\prime}=d \omega / d E$. As $|\gamma|$ increases, the weight of the $J_{r}^{4}$ term increases as well as $\left\langle J_{r}\right\rangle$. However, because the correction has the power $3 / 2$, the deviation with respect to the sphere remains small.

A similar treatment can also be carried ou in the case of oblate top products. However, the result differs because the smaller rotational constants changes from $B \simeq B_{0}(1+4 \gamma / 3)$ for $\gamma<0$ to $B_{s} \simeq B_{0}(1-2 \gamma / 3)$ for $\gamma>0$ :

$$
\begin{aligned}
P\left(J_{r} ; J=0\right) & \simeq 2 J_{r} \int_{B_{0} J_{r}^{2}+a J_{r}^{3}}^{E-E_{0}} \omega\left(E-E_{0}-\varepsilon_{\mathrm{tr}}\right) d \varepsilon_{\mathrm{tr}} \\
& +\frac{4 B_{0}}{3} \gamma J_{r}^{3} \omega\left(E-E_{0}-B_{0} J_{r}^{2}-a J_{r}^{3}\right),
\end{aligned}
$$

The corrective term is still positive, and favors higher values of $J_{r}$ at increasing $\gamma$. However, this term grows linearly with $\gamma$, therefore the correction is more important for oblate tops than for prolate tops. 
The increase in product angular momentum, as well as the non equivalence of the two deformations close to the spherical shape, are indeed seen in Figs. 7)(a,b), and these effects are also observed at nonzero initial angular momenta, Figs. 7l(c,d). Obviously, other parameters to quantify the extent of deformation might affect the shape of the curves $\left\langle J_{r}\right\rangle(\gamma)$ or $\left\langle\varepsilon_{\mathrm{tr}}\right\rangle(\gamma)$. For instance, if $\gamma$ was defined from the inertia momenta or rotational constants directly, $\left\langle J_{r}\right\rangle$ would vary differently around zero, because the inertia momenta vary quadratically with the axes lengths $a$ and $b$.

Increasing $|\gamma|$ tends to favor larger product angular momenta, which is a consequence of smaller rotational constants, the same rotational energy being achieved through higher $J_{r}$. In turn, the centrifugal barrier increases and the kinetic energy released decreases. This explains the correlation between $\left\langle\varepsilon_{\text {tr }}\right\rangle$ and $\left\langle J_{r}\right\rangle$ seen in Figs. [6] and [7 Larger total energies decrease the relative importance of the rotational contribution, as well as the effects of nonsphericity. This is precisely what we observe in Figs. 6(b) and 7(b). Conversely, if now we increase $J$ keeping the total energy fixed, more kinetic energy is allowed in the rotational modes, which usually yields amplified deviations with respect to the spherical reference for both the KER and the product angular momentum.

At finite angular momentum $J$, it is much harder to provide simple explanations for the behavior of the statistical quantities, because new parameters come to play an important role. Increasing $J$ generally has a much more dramatic effect than increasing $E$. The minimum value for the KER, $\varepsilon_{\mathrm{tr}}^{\min }$, has a component which grows linearly with the smallest rotational constant of the product. ${ }^{11,23}$ At large deformations, either prolate or oblate, $\varepsilon_{\text {tr }}^{\min }$ always takes values smaller than for the spherical reference. Therefore low values of the KER are further statistically favored. This effect may be larger than the small increase due to the centrifugal energies seen in Fig. 6(a). Second, because high deformations are characterized with at least one very small rotational constant, the upper limits $J_{r}(Y)$ of the product angular momentum in the $\left(L, J_{r}\right)$ integration range can reach higher values. Thus the integration range extends to larger $J_{r}$, and the average value of $\left\langle J_{r}\right\rangle$ increases.

The competition between these various effects, as well as the different functional forms for the RDOS, provide a rather rich variety of behaviors for $\left\langle\varepsilon_{\mathrm{tr}}\right\rangle$ and $\left\langle J_{r}\right\rangle$, as shown in Figs. 6] and 7 . The exact numerical calculations displayed in Figs. 6] (c,d) and Z7(c,d) confirm that much larger deviations are seen with respect to the spherical reference when $J$ is nonzero. In particular, the marked decreases in the KER and the correlated increases in the product angular momentum are of larger magnitude than for $J=0$. A notable result is that the most important differences between the two types of deformation (oblate and prolate) are exclusively seen at very low total momentum and for large sizes. These differences are related to variations in small rotational constants. Above some specific total energy and/or some total angular momentum, the effects of prolate or oblate deformations become rather similar in shape and magnitude, except very close to $\gamma=0$.

Another interesting feature of these nonspherical systems is their ability to adopt a preferential direction for the rotational excitation following evaporation, as measured with respect to the cylindrical axis. The average cosine of the angle between the product angular momentum and the symmetry axis, as obtained from Eq. (19), is shown in Fig. 8 for the same mechanical conditions as in Figs. 6] and 7 versus the extent of deformation $\gamma$. Again, the effects monotonically decrease with increasing cluster size.

From the previous discussion we already know that $\langle\cos \theta\rangle$ is positive (resp. negative) for oblate (resp. prolate) deformations. Hence, the variations of this quantity seen in Fig. 8 are not surprising, at least for small deformations. A simple, first-order perturbative expansion in the equation (19) can be used as a check of the behavior of $\langle\cos \theta\rangle$ close to $\gamma \sim 0$. However, for arbitrary total energies and angular momenta, the extent of alignment $\langle\cos \theta\rangle$ always shows the same qualitative variations with respect to $\gamma$. For oblate top products, dissociation induces rotation around a long axis preferentially, and the effect is more significant at smaller total energies or larger initial angular momenta. Prolate top products, on the other hand, display a non-monotonic behavior, concomitant with the variations of the large rotational constant, Eq. (22), which has a maximum when $\gamma=(\sqrt{2}-1) /(\sqrt{2}+1)$. The fact that alignment is hindered at very large oblate deformations is a simple consequence that the two rotational constants $B$ and $C$ tend to 0 , hence the second integral over $L$ and $J_{r}$ in Eq. (19) goes to 0, and the system becomes equivalent to a sphere again from the point of view of its orientation. Therefore, it is practically impossible to reach a perfect statistical alignment for oblately deformed systems. Such a situation is never met with prolate deformed clusters, since one rotational constant always keeps increasing for large deformations. The shape of the curves in Figs. 8) (c,d) also reveals that alignment can be quite effective, even at moderate deformations. For a 50-atom cluster, $J \sim 20$ is close to the typical thermal angular momentum near the melting point, and a deformation of about $20 \%$ is enough to induce statistical alignment beyond $25 \%$.

Finally, following our previous work, ${ }^{23}$ we focus on the influence of nonsphericity on the rotational cooling and heating effects. The same simple model for LJ clusters was used in the harmonic approximation for the VDOS and the $C / r^{6}$ radial potential for the dissociation energy. We consider here more realistic conditions for dissociation, namely thermal distributions for the energy and angular momentum of the parent cluster, as well as a possible extra energy shift to model an initial, brief excitation (from photoabsorption or collision). To provide ground for comparison, these distributions are assumed to be Boltzmann-like and identical for all geometries. The final distributions were calculated by taking a full account of the prolate or oblate character of the main product cluster. 
In spherical clusters, we have seen that a purely thermal distribution generally leads to rotational cooling, i.e. the distribution of angular momentum of the product cluster is shifted to lower values ${ }^{23}$ On the other hand, an initially cold cluster submitted to a sudden vibrational excitation preferentially exhibits rotational heating ${ }^{23.26}$ In Fig. 9 the final angular momentum distributions are compared for the three types of products. Even though the product clusters are significantly deformed, the general rotational behavior remains similar to that of the spherical top. As seen from Fig. 9(a), purely thermal evaporation induces rotational cooling, and this effect is attenuated for nonspherical clusters. Deformation also influences the final distribution itself, which differs significantly from the Boltzmann behavior for prolate systems. These results are consistent with our previous analysis, as thermal evaporation corresponds more likely to the cases (c) and (d) of Figs. 66 8 with substantial initial $J$. At $|\gamma|=0.5$, Fig. [7 (d) shows that $\left\langle J_{r}\right\rangle$ increases by about $5 \%$ for oblate top products, slightly less for prolate top products, with respect to the spherical case. These changes (including their magnitude) are reflected on the distributions in Fig. 9(a).

Rotational heating is produced in a different way, by adding a sharp extra vibrational excitation to an initially cold cluster. Deformed clusters also exhibit rotational heating, as shown in Fig. 9(b). For both prolate and oblate top products, deformation further heats the final rotational motion, but the two deformations behave dissimilarly, the effect being much stronger for the oblate top product. This is understood by considering again Fig. Z7(b), which shares the mechanical conditions of high total energies but low angular momenta. The increase in $\left\langle J_{r}\right\rangle$ is quite important for oblate tops, although it is barely noticeable for prolate tops at $|\gamma|=0.5$.

Deformation most often induces higher rotational excitations upon evaporation, this explains why it reduces rotational cooling, but enhances rotational heating.

\section{CONCLUSION}

The present work was aimed at providing a quantitative study of the effects of cluster shape on its evaporation statistics. Within the framework of phase space theory, the distributions of kinetic energy released and final angular momenta were obtained assuming the fragments could be described as an atom plus a prolate or an oblate top as the main product.

These theoretical tools were challenged on the example of the unimolecular dissociation of the 8-atom LennardJones cluster, chosen for its significantly oblate shape. The small disagreement reported earlier ${ }^{22}$ between PST and molecular dynamics simulations for the distribution of final angular momentum was solved after accounting for the nonspherical character of $\mathrm{LJ}_{7}$.

Using simple models for the vibrational density of states and the dissociation potential, we systematically investigated the changes induced by deformation. We found very significant deviations with respect to the spherical case for both the final angular momentum and kinetic energy, especially for nonzero initial momenta. In such cases, the relative variations of the energetic properties are larger than those of angular momenta by up to even one order of magnitude.

We could interpret these changes as originating partly from the specific centrifugal barrier in our present implementation of PST, but also from the new energy constraints arising with lower rotational constants. These effects are not included in simpler statistical rate theories of unimolecular dissociation such as the Klots models, not mentioning the Engelking-Weisskopf $\mathrm{f}^{29.30}$ or RRK approaches $\stackrel{31}{\underline{31}}$

We also obtained information about the relative orientation of the product angular momentum, by considering restricted integration of the rotational densities of states. Alignment toward the long axes is generally observed for all deformations, in agreement with mechanical arguments. This alignment was seen to be very effficient already at moderate initial angular momenta, and for moderate deformations.

Finally, we discussed the influence of deformation on the rotational cooling and heating effects resulting from specific excitations. While cooling is attenuated for nonspherical clusters, heating is amplified. These behaviors are attributed to the decrease of one rotational constant, which favors larger angular momenta in the product cluster.

The examples considered here were obtained on model clusters, but we expect the present results to be relevant for more realistic systems. Charged rare-gas clusters, which display very prolate shapes below 15 atoms due to the presence of a linear ionic trimer, have been the subject of intense experimental and theoretical activity from the point of view of their dissociation patterns 27

Polycyclic aromatic hydrocarbon (PAH) molecules are strongly oblate and their rotation was considered within the astrophysical context by Rouan and co-workers ${ }^{32}$ Dehydrogenation of these molecules, in particular, has received a special attention among several experimental and theoretical groups ${ }^{33.34 .35}$ This problem could be investigated with the same methods used here.

In these two examples, a realistic atomistic description requires models much more involved than simple pairwise potentials. This practical limitation prevents one fully relying on brute-force simulations, thus making statistical approaches most useful in the future. 


\section{Acknowledgments}

The authors wish to thank the GDR Agrégats, Dynamique et Réactivité for financial support.

${ }^{1}$ W. Forst, Unimolecular Reactions (Cambridge University Press, Cambridge, 2003).

2 R. G. Gilbert and S. C. Smith, Theory of Unimolecular and Recombination Reactions (Blackwell Scientific, Oxford, 1990).

${ }^{3}$ M. F. Jarrold, Introduction to statistical reaction theories, in Clusters of Atoms and Molecules I, edited by H. Haberland (Springer, Berlin), 1991.

4 C. Bréchignac, Ph. Cahuzac, J. Leygnier, J. Weiner, J. Chem. Phys. 90, 1492 (1989).

${ }^{5}$ M. Vogel, K. Hansen, A. Herlert, L. Schweikhard, Appl. Phys. B 73, 411 (2001).

${ }^{6}$ S. Weerasinghe, F. G. Amar, Z. Phys. D: At., Mol. Clusters 20, 167 (1991); J. Chem. Phys. 98, 4967 (1993).

7 G. H. Peslherbe and W. L. Hase, J. Phys. Chem. A 104, 10556 (2000).

8 E. Nikitin, Theor. Exp. Chem. 1, 83 (1965); 1, 90 (1965).

9 P. Pechukas and J. C. Light, J. Chem. Phys. 42, 3281 (1965).

10 C. E. Klots, J. Phys. Chem. 75, 1526 (1971).

11 W. J. Chesnavich and M. T. Bowers, J. Chem. Phys. 66, 2306 (1977).

12 M. F. Jarrold and V. A. Constant, Phys. Rev. Lett. 67, 2994 (1991).

13 Ph. Dugourd, R. R. Hudgins, J. M. Tenenbaum, and M. F. Jarrold, Phys. Rev. Lett. 80, 4197 (1998).

14 A. A. Shvartsburg and M. F. Jarrold, Phys. Rev. A 60, 1235 (1999).

15 F. Calvo, S. Tran, S. A. Blundell, C. Guet, and F. Spiegelman, Phys. Rev. B 62, 10394 (2000) and references therein.

16 U. Näher, S. Bjørnholm, S. Frauendorf, F. Garcias, and C. Guet, Phys. Rep. 285, 245 (1997).

17 M. Berblinger and C. Schlier, J. Chem. Phys. 96, 6834 (1992).

18 W. J. Chesnavich and M. T. Bowers, Prog. React. Kinet. 11, 137 (1982).

19 D. H. Li and J. Jellinek, Z. Phys. D: At. Mol. Clusters 12, 177 (1989).

20 M. A. Miller and D. J. Wales, Mol. Phys. 89, 533 (1996).

21 F. Calvo and P. Labastie, Euro. Phys. J. D 3, 229 (1998).

22 F. Calvo and P. Parneix, J. Chem. Phys. 119, 256 (2003).

23 P. Parneix and F. Calvo, J. Chem. Phys. 119, 9469 (2003).

24 F. Calvo and P. Parneix, J. Chem. Phys. 120, 2780 (2004).

25 P. Parneix, Ph. Bréchignac, and F. Calvo, Chem. Phys. Lett. 381, 471 (2003).

26 A. J. Stace, J. Chem. Phys. 93, 6502 (1991).

27 see F. Calvo, J. Galindez and F.-X. Gadéa, Phys. Chem. Chem. Phys. 5, 321 (2003) and references therein.

28 H. Goldstein, Classical Mechanics, 2nd edtion (Addison-Wesley, Reading, 1980).

29 P. C. Engelking, J. Chem. Phys. 85, 3103 (1986); 87, 936 (1987).

${ }^{30}$ V. Weisskopf, Phys. Rev. 52, 295 (1937).

31 O. K. Rice and H. C. Ramsperger, J. Am. Chem. Soc. 50, 617 (1928); L. S. Kassel, J. Phys. Chem. 32, 225 (1928).

32 D. Rouan, A. Léger, A. Omont, and M. Giard, Astron. Astrophys. 253, 498 (1992).

33 Y. Ho, R. Dunbar, C. Lifshitz, J. Am. Chem. Soc. 117, 6504 (1995).

34 P. Boissel, P. Parseval, P. Marty, G. Lefevre, J. Chem. Phys. 106, 4973 (1997).

35 M. Dibben, D. Kage, J. Szczepanski, J. Eyler, M. Vala, J. Phys. Chem. A 105, 6024 (2001). 


\begin{tabular}{|l|l|l|l|l|l|l|l|l|}
\hline & \multicolumn{2}{|c|}{ spherical } & \multicolumn{2}{|c|}{ oblate } & \multicolumn{2}{c|}{ prolate } & \multicolumn{2}{c|}{ MD } \\
\hline & $\left\langle\varepsilon_{\text {tr }}\right\rangle$ & $\left\langle J_{r}\right\rangle$ & $\left\langle\varepsilon_{\text {tr }}\right\rangle$ & $\left\langle J_{r}\right\rangle$ & $\left\langle\varepsilon_{\text {tr }}\right\rangle$ & $\left\langle J_{r}\right\rangle$ & $\left\langle\varepsilon_{\text {tr }}\right\rangle$ & $\left\langle J_{r}\right\rangle$ \\
\hline$J=0$ & 0.51 & 1.07 & 0.51 & 1.16 & 0.51 & 1.12 & 0.50 & 1.11 \\
\hline$J=1$ & 0.56 & 1.21 & 0.56 & 1.31 & 0.56 & 1.27 & 0.55 & 1.25 \\
\hline$J=2$ & 0.70 & 1.45 & 0.70 & 1.58 & 0.69 & 1.52 & 0.68 & 1.52 \\
\hline$J=3$ & 0.89 & 1.65 & 0.89 & 1.85 & 0.87 & 1.80 & 0.89 & 1.84 \\
\hline
\end{tabular}

TABLE I: Average kinetic energy released $\left\langle\varepsilon_{\operatorname{tr}}\right\rangle$ and product angular momentum $\left\langle J_{r}\right\rangle$ after evaporation of the LJ $\mathrm{J}_{8}$ cluster at total excitation energy $E / n=1.2$ and several total angular momenta. The values obtained from molecular dynamics simulations are compared to the PST predictions in the spherical $(\gamma=0)$, prolate top $(\gamma=-0.3)$ and oblate top $(\gamma=0.3)$ approximations for the product cluster $\mathrm{LJ}_{7}$. 

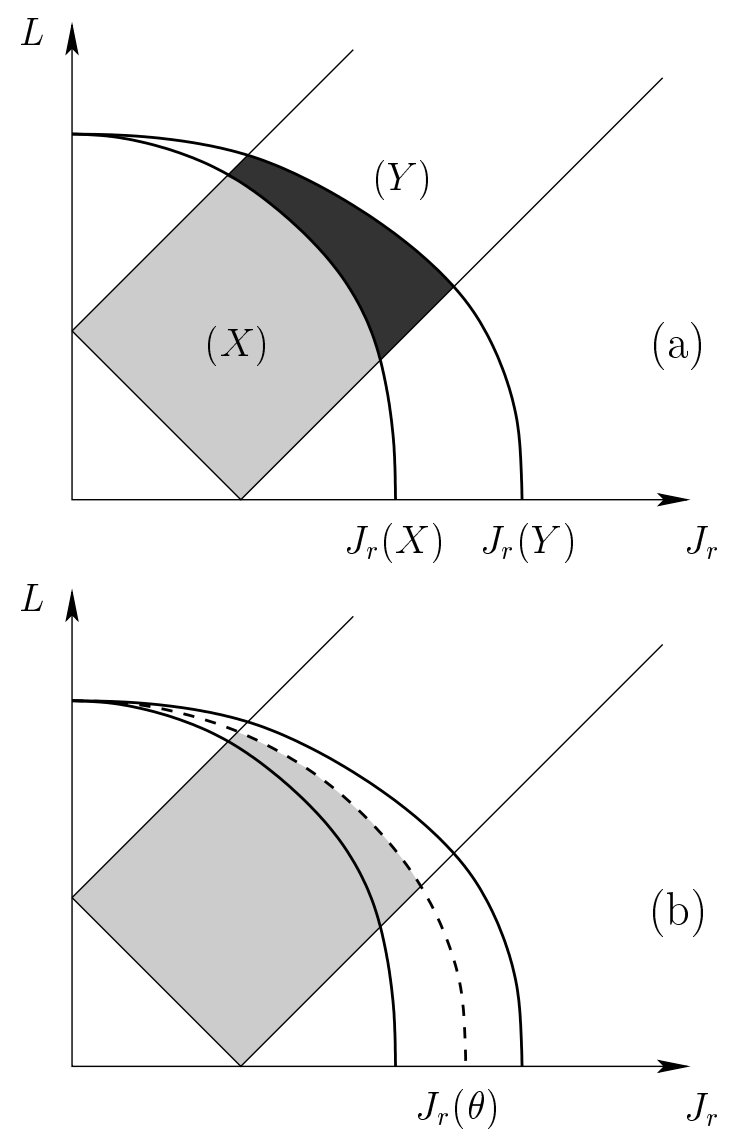

FIG. 1: Range of integration in the $\left(L, J_{r}\right)$ plane for atom+symetric top fragments. (a) Complete integration range, $X>Y$ being the two rotational constants. (b) Restricted integration range when the orientation of $J_{r}$ with respect to the symmetry axis is restricted to have a fixed angle $\theta$.

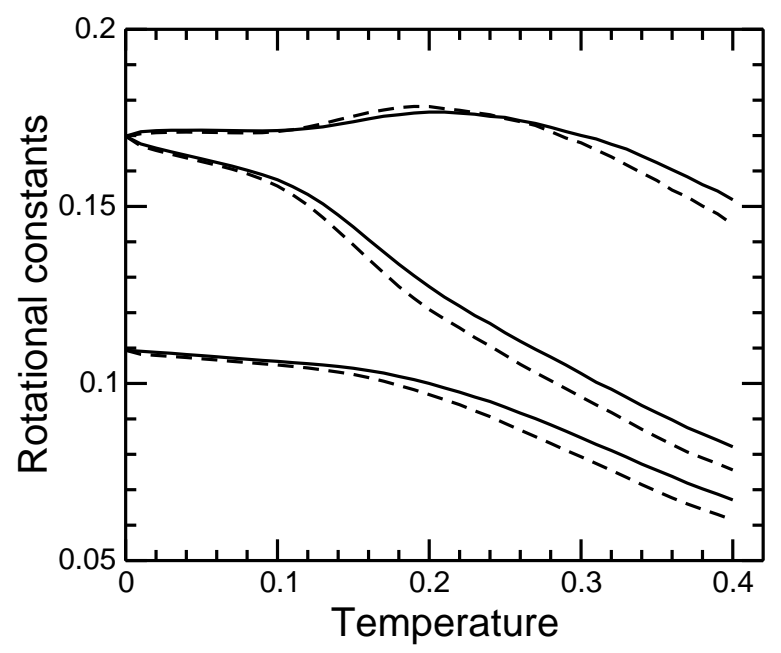

FIG. 2: Thermally averaged rotational constants of the $\mathrm{LJ}_{7}$ cluster versus temperature, for initially nonrotating (solid lines) and rotating $(J=3$, dashed lines) systems. 


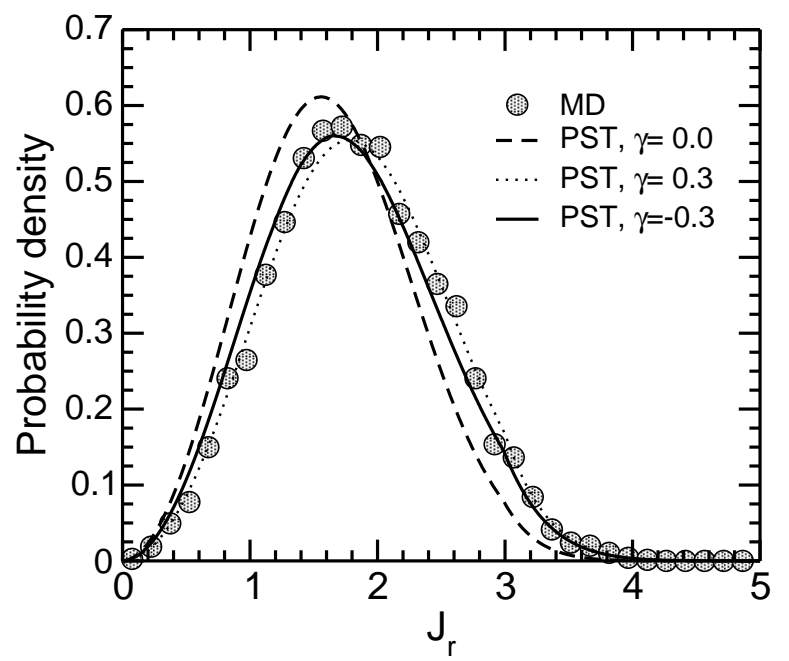

FIG. 3: Distribution of final angular momentum after dissociation of $\mathrm{LJ}_{8}$, from different approximations (sphere-atom, prolateatom and oblate-atom) for the rotational DOS in the PST calculation.

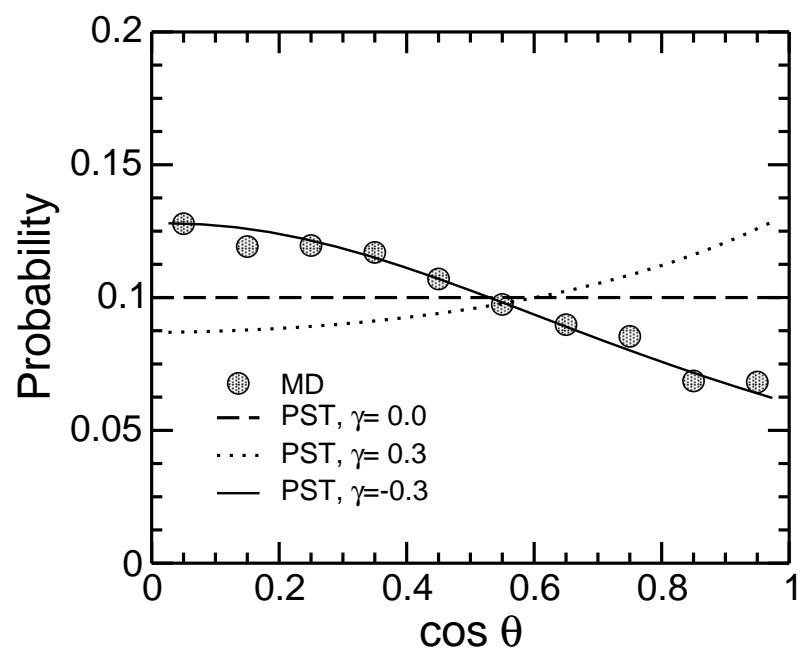

FIG. 4: Distribution of $\cos \theta$ in the dissociation of $\mathrm{LJ}_{8}$ at $J=3$ and $E / n=1.2$. The results of MD simulations are compared to PST calculations for different approximations of the $\mathrm{LJ}_{7}$ main product. 


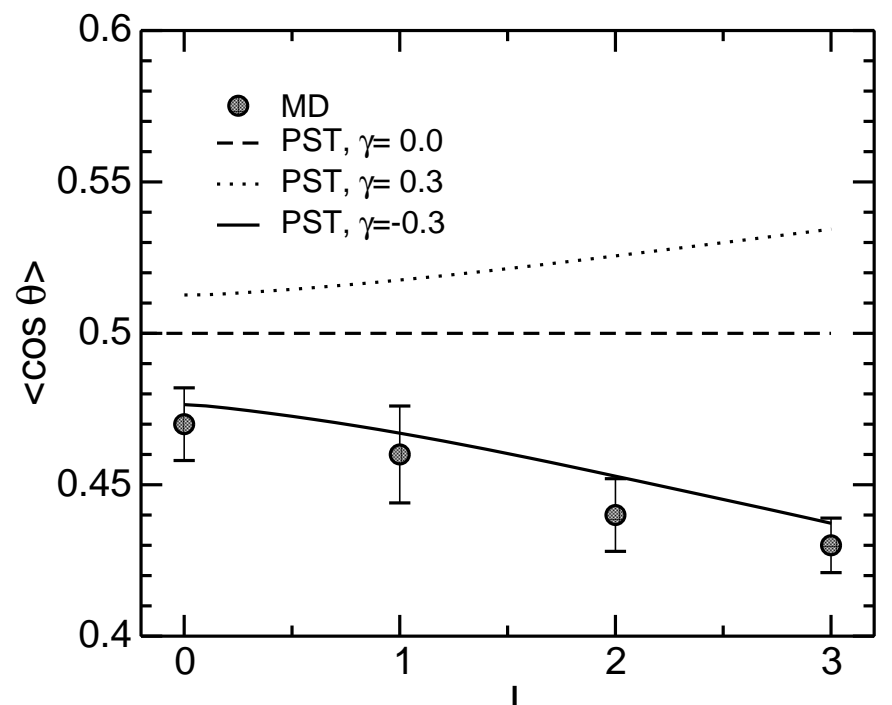

FIG. 5: Average angle $\langle\cos \theta\rangle$ between the symmetry axis and the final angular momentum versus initial $J$ in the dissociation of $\mathrm{LJ}_{8}$ with $E / n=1.2$.
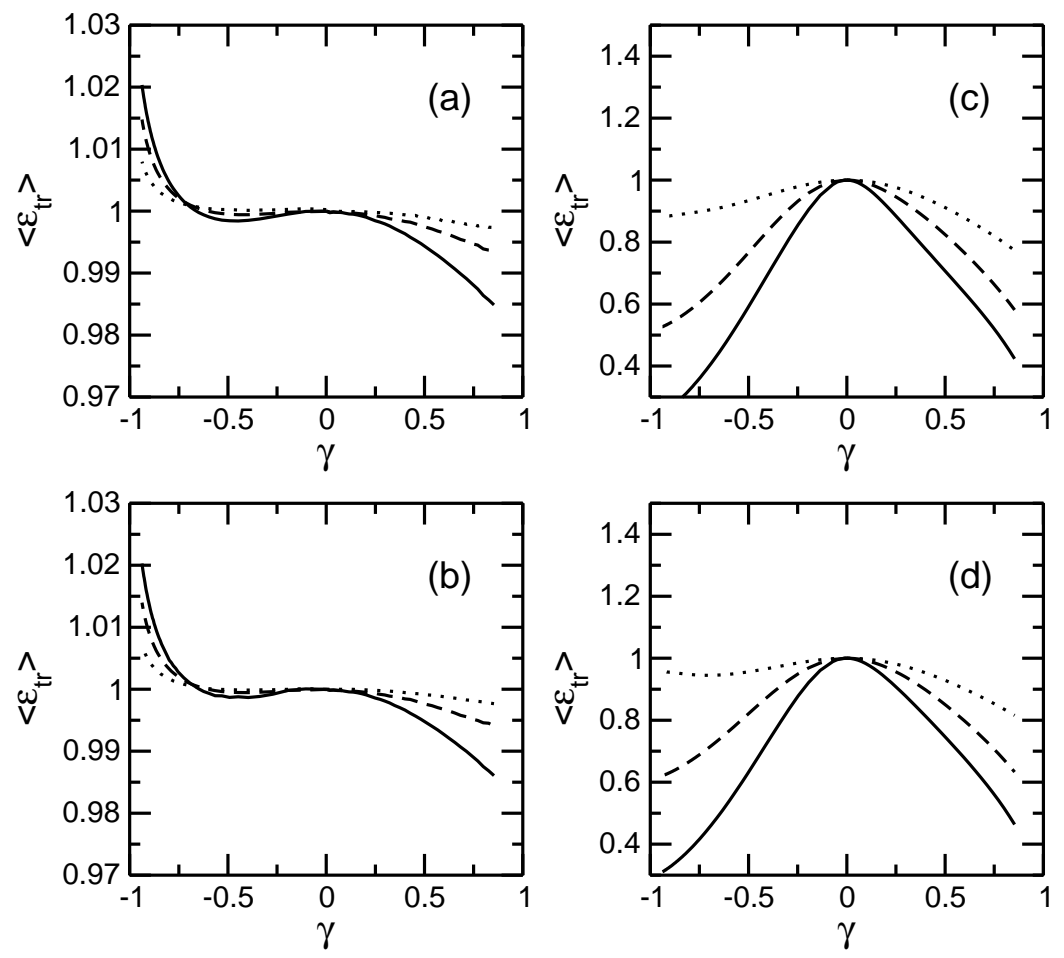

FIG. 6: Average KER $\left\langle\varepsilon_{\mathrm{tr}}\right\rangle$ versus deformation $\gamma$ in the evaporation of model $\mathrm{LJ}_{n+1}$ clusters under several conditions. (a) $E / n=1.2$ and $J=0$; (b) $E / n=1.2$ and $J=20$; (c) $E / n=0.9$ and $J=0$; and (d) $E / n=0.9$ and $J=20$. The results are shown for $n=50$ (solid lines), $n=100$ (dashed lines), and $n=200$ (dotted lines). 

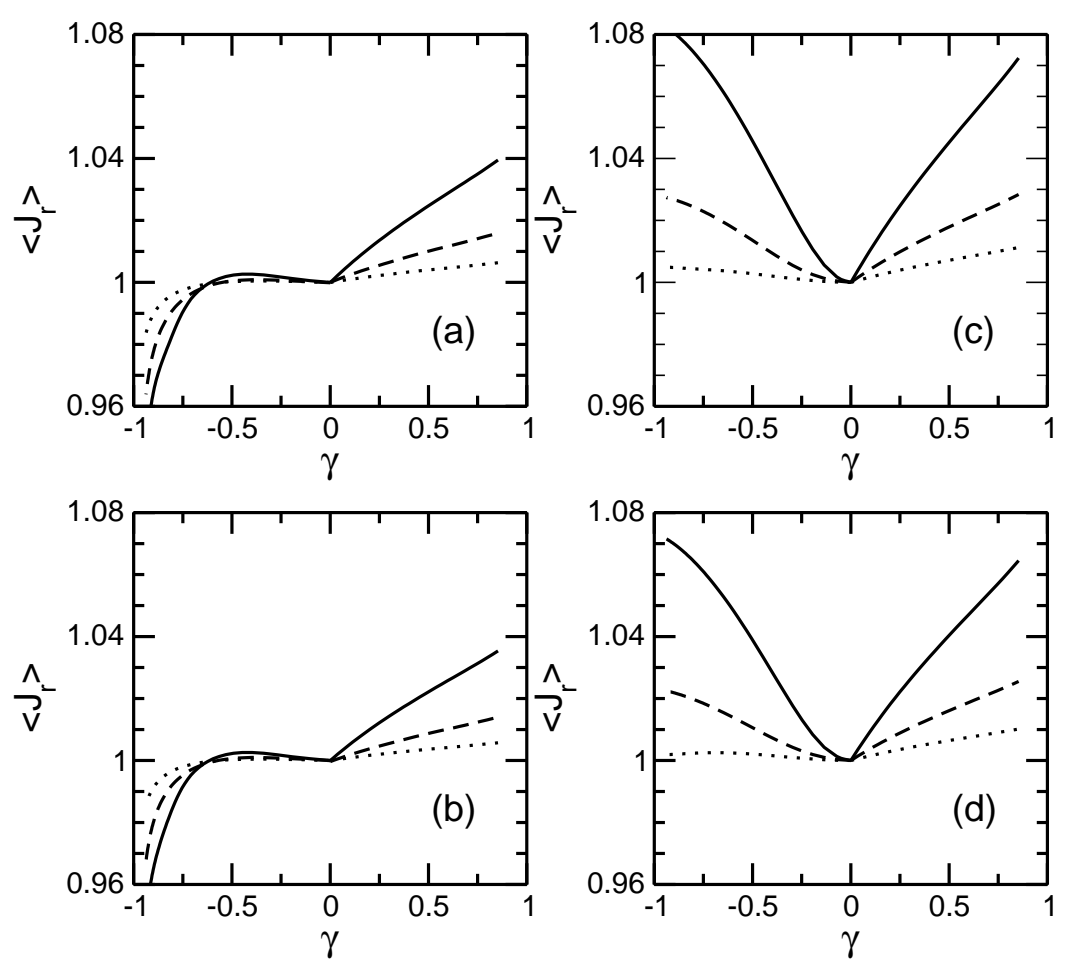

FIG. 7: Average product angular momentum $\left\langle J_{r}\right\rangle$ versus deformation $\gamma$ in the evaporation of model LJ $J_{n+1}$ clusters under the same conditions as in Fig. [6] The same graphs conventions are also used.
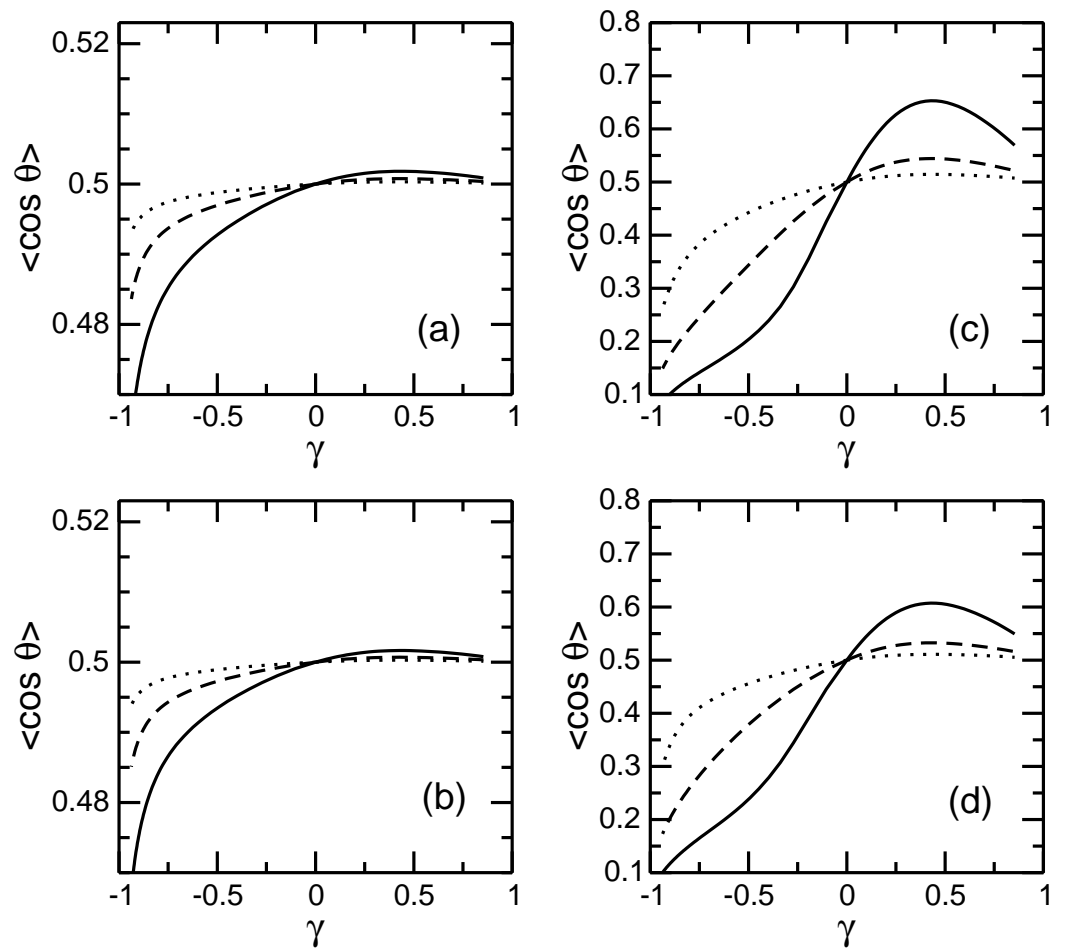

FIG. 8: Average orientation $\langle\cos \theta\rangle$ between the symmetry axis and the product angular momentum vector versus deformation $\gamma$ in the evaporation of model $\mathrm{LJ}_{n+1}$ clusters under the same conditions as in Fig. 6] The same graphs conventions are also used. 

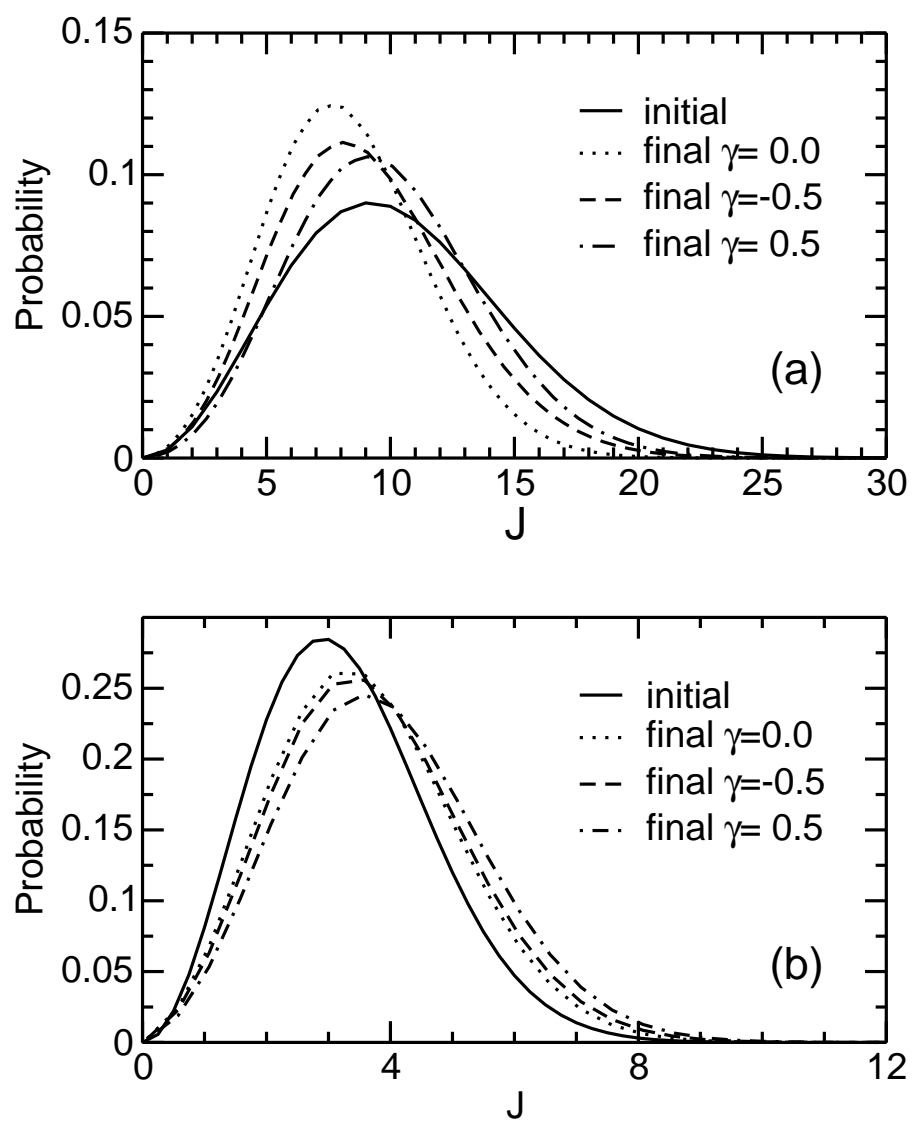

FIG. 9: Initial and final distributions of angular momentum after evaporation from a 51-atom model LJ cluster, the main product being assumed spherical $(\gamma=0.0)$, prolate $(\gamma=-0.5)$, or oblate $(\gamma=0.5)$. (a) Hot thermal distributions of energy and angular momentum $(T=0.5)$; (b) cold thermal distributions $(T=0.05)$ and extra vibrational excitation $(\Delta E=30)$. 